\title{
Improvement Algorithm of Background Updating Based on Kalman Filter Jin Wang ${ }^{1, a^{*}}$ and Hang Zhou ${ }^{1, b}$ \\ ${ }^{1}$ School of Electronic and Information Engineering, Beijing Jiaotong University, Beijing 100044, China \\ ajinwang@bjtu.edu.cn, bhangzhou@bjtu.edu.cn
}

Keywords: Target detection; Mix-color space; Background updating; Kalman filter

\begin{abstract}
In the process of target detection under complex background, the color of target (foreground) is often similar to the color of the background which causes noise during background updating, and the integrity of the target extracted from the image has been affected. In view of this situation, an improved algorithm based on Kalman filter is proposed. The algorithm analyzes the difference of background subtraction results in different channels of multiple color spaces, then the mix-color space is built and the detection results of each channel in the mix-color space are integrated. The background updating algorithm based on Kalman filter has been improved from the update region and the updated background was closer to real background. The experiment results show that under the complex background, the algorithm can remove the background noise effectively with high accuracy and good applicability.
\end{abstract}

\section{Introduction}

In recent years, target detection has become a hot issue in computer vision. It has an extensive application prospect in security monitoring and intelligent transportation.

There are 3 major common methods. Optical flow method [1], background difference method [2] and frame difference method [3] are common algorithms of target detection. The moving objects can be detected by Optical flow method without knowing any information of scene, but it is not suitable for real-time processing. The inter-frame difference method has the performance of simple implementation and low complexity, its disadvantages that it cannot extract the complete target. Being sensitive to ambient noise is the shortcoming of frame difference, which is overcame by background difference method. Compared with the optical flow method, the background difference method has lower computational cost and lower noise sensitivity for the requirement of real-time detection. The background cannot be immutable in the implementation of the background difference method. Therefore, a background update model should be added to ensure that the background image can change with the environment changes.

Jesse Scott et al. [4] proposed a background update algorithm based on Kalman filter which estimates the changes of background in real-time and effectively reduce error of target detection. Ji et al. [5] combine the motion area from four frame difference algorithm with moving target determined by Surendra background difference method to get the moving area image, the real-time background model can effectively established. That method can adapt to the sudden illumination very well and determine the threshold quickly. As a result, the accuracy of target detection was improved.

The background update algorithm is analyzed from different angles through above algorithms. All of them have achieved good results, but they ignored the research on multiple color spaces while focusing on a single color space. Compared with the single color space, multiple color spaces have a larger amount of information.

Therefore, this paper detects targets in multiple color spaces and builds the mix-color space by analyzing the complementarity of detection results in different channels. On this basis, an improved background updating algorithm based on Kalman filter is proposed to deal with the problem that the color of human body is similar to the background. 


\section{Proposed Algorithm}

Background Difference Algorithm Based on Mix-color Space. In this paper, we use the background difference method and the process of it can be presented by Eq. (1) in which $I(i, j, k)$ represents the image at time $k, B(i, j, k)$ represents the background image at time $k, \Delta I$ represents the foreground image obtained by the background difference method.

$$
\Delta I(i, j, k)=I(i, j, k)-B(i, j, k)
$$

The single-channel background difference results in mix-color space are integrated in this paper. The mix-color space [6] is a color space obtained by reassembling three channels which have better detection results than others. Color space is usually described by three relatively independent channels where different channels can represent different color characteristic, detection results are also different in different color space as a result [7]. RGB, $\mathrm{HSV}, \mathrm{YC}_{\mathrm{b}} \mathrm{C}_{\mathrm{r}}$, YUV, etc. are widely used currently. The re-combination in this paper refers that the three best channels detection results are combined by OR operation. As shown in Eq. (2), where $p$ represents the three best color channels in mix-color space, $\Delta I$ represents the foreground image which integrated by the detection results of the three best channels.

$$
\Delta I(i, j, k)=\sum_{p=1}^{3} \Delta I_{p}(i, j, k)
$$

In order to analyze and compare the detection results of each color channel, the differential operation between each channel and the corresponding channel of background image is calculated. The mixed color space can be established with the three channels which can get a more complete target after integrating by observing and analyzing the detection results.

Improvement Algorithm of Background Updating Based on Kalman Filter. This paper assumes that the camera is stationary, human motion environment is not dramatic changes and the human will not stay in the same position for a long time. In this case, the paper uses the median method for background modeling [8].

Firstly, the foreground image of the initial frame is obtained by the background difference method. In order to eliminate the influence of illumination and scene changes on the human body, the background needs to be updated in real time. Background updating algorithm based on Kalman filter [9] is applicable to situation that the changes of moving target is much larger than the background and it often used to solve the problem of background updating. The slowly changing background image is predicted by time-domain recursive of Kalman filtering theory, $B(i, j, k+1)$ is the result of the previous state prediction. The images were divided into two parts: the stationary background and the foreground include the moving human body, and then a more accurate background model can be built through weighted updating of the two parts.

However, the updated background images remain some of foreground images because of the holes in the detected foreground regions. In this paper, the background algorithm of Kalman filter is improved based on mix-color space and the background update region is adjusted. The background and foreground integrated by the results of three channels in mix-color space are used to make a recursive prediction and update the background to reduce background residual noise. The improved algorithm is shown as follows.

$$
\begin{aligned}
& B(i, j, k+1)=B(i, j, k)+g(k) *(I(i, j, k)-B(i, j, k)) \\
& g(k)=\beta^{*}(1-M(i, j, k))+\alpha^{*} M(i, j, k) \\
& M_{p}(i, j, k)= \begin{cases}1 & |I(i, j, k)-B(i, j, k)|>T h \\
0 & \text { otherwise }\end{cases} \\
& M(i, j, k)=\sum_{p=1}^{3} M_{p}(i, j, k)
\end{aligned}
$$


$B(i, j, k+1)$ represents the background image at time $k+1, \alpha$ is the motion factor, $\beta$ is the background factor, and the adaptive parameters $\alpha$ and $\beta$ follow the Gauss distributions. Th represents the threshold value, and is obtained by OTSU algorithm. $M(i, j, k)$ represents the value integrated by the three color channels. The value obtained by $M(i, j, k)$ is substituted into Eq. (3) and (4), then the process of an improved background updating is made.

Compared with the traditional Kalman filter background updating algorithm, the improved algorithm can eliminate the foreground noise in the background image.

\section{Experimental Results}

The experiment was done under VS 2010 and OpenCV2.4.9. The hardware environment is a PC with $2.80 \mathrm{GHz}$ CPU, 2.00GB memory, Windows 7 Professional 32-bit operating system. In this paper, CASIA gait database Dataset A was selected. This paper is based on the image sequences which with 0 degree of the image plane. Five sequences (hy, zjg, ljg, rj, wl) of pictures were selected for evaluation of the algorithm, which included the sequence that foreground color is close to the background. The total number of the test images is 192 ; each size is $352 \times 240$.

Establishment of Mixed Color Space. In this paper, RGB, HSV and $\mathrm{YC}_{b} \mathrm{C}_{r}$ are chosen to analyze and establish mixed color space. In the process of the image sequence, the original image comes from RGB and $\mathrm{HSV}, \mathrm{YC}_{\mathrm{b}} \mathrm{C}_{\mathrm{r}}$ format need a conversion.

The image frame and the background frame are decomposed into 9 color channels: R, G, B, H, S, $\mathrm{V}, \mathrm{Y}, \mathrm{C}_{\mathrm{b}}$ and $\mathrm{C}_{\mathrm{r}}$. Then the foreground region is obtained by background difference and binarization. Fig. 1 is a frame image where the color of its foreground is close to backgroundimage, Fig. 3 shows the experimental results of the image, in which the white area is the detected foreground area. It can be seen from Fig. 3 that when the background and the foreground's color are close, the single-channel background difference method is difficult to get the complete information, but the foreground target information obtained by different channels can be complementary.

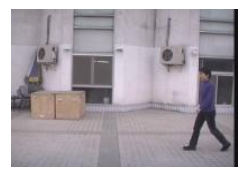

Figure 1. Original image

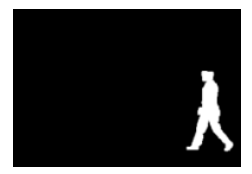

Figure 2. Human detection result in mixed color space

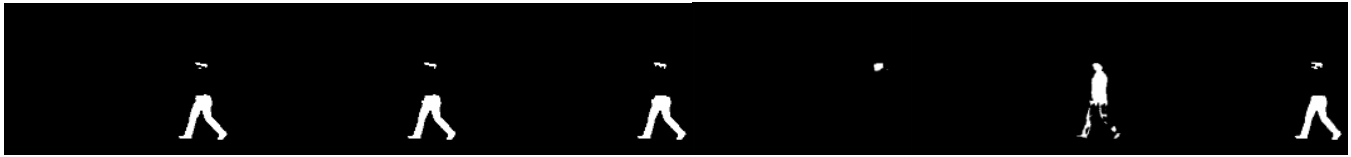

(a)R channel (b) G channel (c) B channel (d) H channel (e) S channel (f) V channel

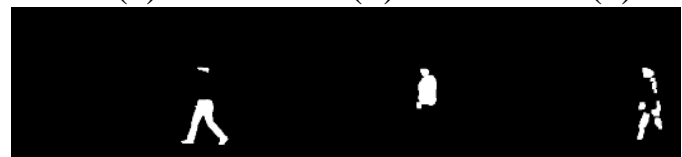

(g) Y channel (h) $\mathrm{C}_{\mathrm{b}}$ channel (i) $\mathrm{C}_{\mathrm{r}}$ channel

Figure 3. Binarization of foreground area in each channel

From experimental images, it was found that $G 、 S 、 C_{r}$ channels could be combined to achieve complementary effects. Therefore, G, S, and $\mathrm{Cr}$ were chosen as three channels of mix-color space. The results after mixing and morphological processing are shown in Fig. 2.

From the results above, we can see that a better test results can be obtained when the foreground color is close to background. After the experiment with the test images whose total number was 192 , the results showed that the accuracy of the binary image compared with the real image was $91 \%$.

Background Updating. In order to improve the effectiveness of the proposed algorithm, the Kalman background update algorithm and the algorithm with excellent performance of reference [5] were used to detect the test image and compare it with proposed algorithm. From Fig. 4 (b), (c) and 
(d), we can see that when the moving target and the background color are very close, the algorithm [5] is more effective than the Kalman background updating algorithm, but there was still some noise on the updated background. And the update result of the proposed algorithm was more close to the current background, and the residual noise was less than the former two algorithms.

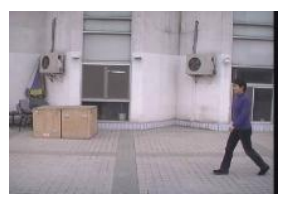

(a) Original image

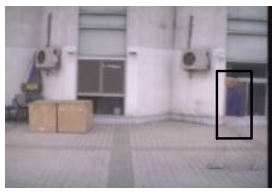

(b)Kalman

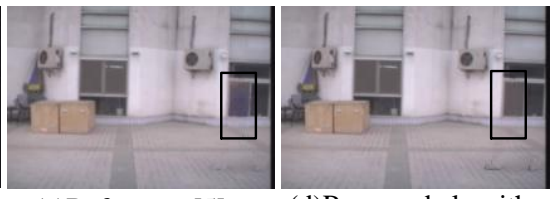

(c)Reference [5]
(d)Proposed algorithm

Figure 4. Images comparison before and after improvement

The initial frame is regarded as the standard image so as to analyze the performance of algorithms objectively. The SNR [10] of the updated background images based on Kalman filter, the algorithm of reference [5] and the proposed algorithm in this paper compared with the standard image are calculated respectively. The results are shown in Fig.5. The SNR of the improved background images obtained by the proposed algorithm is obviously improved compared with the background images based on Kalman filter, and is higher than those of the reference [5]. In order to show the improvement of SNR clearly, average value of three algorithms are listed in Table 1. It can be found that SNR of the algorithm in reference [5] and this paper are all improved, but the SNR of the proposed algorithm is higher than that of the reference [5], which shows that the proposed algorithm is more effective than the reference [5] and the quality of the background images are improved.

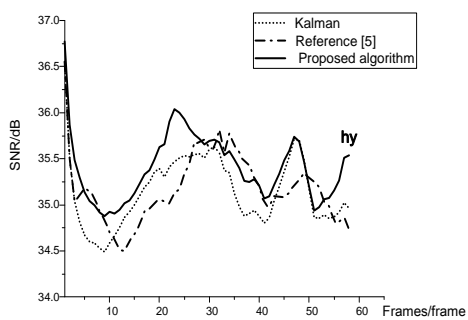

(a) hy

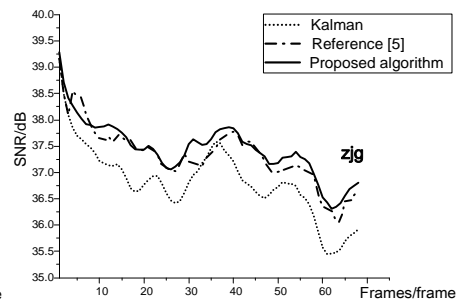

(b)zjg

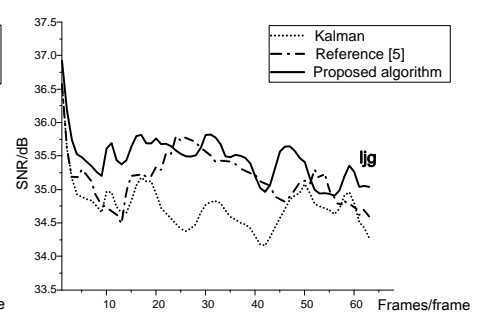

(c)ljg

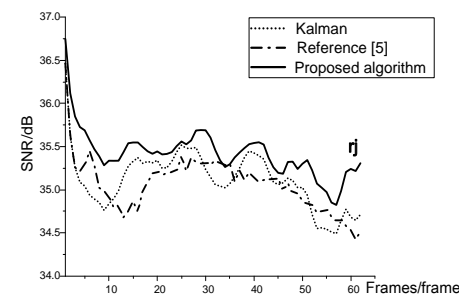

(d)rj

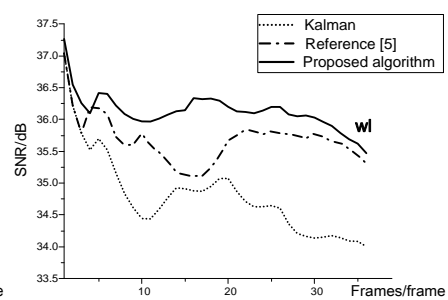

(e)wl

Figure 5. SNR graph

It can be seen that the algorithm proposed in this paper solves the problem that some points of the foreground is misjudged as the background points when the background color is to the foreground, and most of the foreground points remained in the updated background are reduced. Good detection results can be reached by this background update algorithm in this paper when it is used to detect objects with complex background. The algorithm has strong adaptability to the external environment, low noise and high efficiency. 
Table 1 SNR Comparison of Different Algorithms

\begin{tabular}{cccc}
\hline $\begin{array}{c}\text { Image } \\
\text { sequence }\end{array}$ & $\begin{array}{c}\text { Average SNR of } \\
\text { Kalman }\end{array}$ & $\begin{array}{c}\text { Average SNR of } \\
\text { reference[5] }\end{array}$ & $\begin{array}{c}\text { Average SNR of } \\
\text { proposed algorithm }\end{array}$ \\
\hline hy & $35.14 \mathrm{~dB}$ & $35.17 \mathrm{~dB}$ & $35.40 \mathrm{~dB}$ \\
zjg & $36.82 \mathrm{~dB}$ & $37.33 \mathrm{~dB}$ & $37.45 \mathrm{~dB}$ \\
ljg & $34.74 \mathrm{~dB}$ & $35.17 \mathrm{~dB}$ & $35.45 \mathrm{~dB}$ \\
rj & $35.11 \mathrm{~dB}$ & $35.06 \mathrm{~dB}$ & $35.42 \mathrm{~dB}$ \\
wl & $34.80 \mathrm{~dB}$ & $35.68 \mathrm{~dB}$ & $36.13 \mathrm{~dB}$ \\
\hline
\end{tabular}

\section{Summary}

Combined with the classical mix-color space, an improved algorithm based on the Kalman background updating algorithm is proposed in this paper, which solves the problem of background noise when the foreground and background color are close to each other. This method inherits the advantages of Kalman background updating algorithm which can update the background in real time. Experimental results show that the proposed algorithm improves the accuracy of background updating.

\section{Acknowledgements}

This work was supported by the National Natural Science Foundation of China (No. 60972093, No. 61271305), School Foundation of Beijing Jiaotong University under Grant No.2010JBZ010.

\section{References}

[1] T. Senst, R. H. Evangelio and T. Sikora. Detecting people carrying objects based on an optical flow motion model, Applications of Computer Vision (WACV), 2011 IEEE Workshop on, Kona, HI, 2011, pp. 301-306.

[2] S. S. Mohamed, N. M. Tahir and R. Adnan. Background modelling and background subtraction performance for object detection, Signal Processing and Its Applications (CSPA), 2010 6th International Colloquium on, Mallaca City, 2010, pp. 1-6.

[3] M. Weng, G. Huang and X. Da,A new interframe difference algorithm for moving target detection, Image and Signal Processing (CISP), 2010 3rd International Congress on, Yantai, 2010, pp. 285-289.

[4] J. Scott, M. A. Pusateri and D. Cornish, Kalman filter based video background estimation, 2009 IEEE Applied Imagery Pattern Recognition Workshop (AIPR 2009), Washington, DC, 2009, pp. $1-7$.

[5] Q.H. Ji, S.P. Yu. Object Detection Algorithm Based on Surendra Background Subtraction and Four-frame Differenc[J].Computer Applications and Software, Vol. 31 (2014) No. 12, pp.242-244. (In Chinese)

[6] K.Q. Ren, P.H. Zhang, H.L. Luo. Algorithm of Moving Target Detection Based on Multiple Information Merging in Hybrid Color Space [J]. Opto-Electronic Engineering, Vol. 41 (2014) No. 4, pp.21-29. (In Chinese)

[7] S.H. Teng, Y.P. Shen. Application Analysis of Color Spaces in Image Colorization [J]. Computer Science, Vol. 36 (2009) No.10, pp.277-279. (In Chinese) 
[8] C.D. Wu, M.X. Li, M. Liu et al. Adaptive Motion Detection Based on Median Background Model [J]. Journal of Shenyang Jianzhu University (Natural Science), Vol. 24 (2008) No. 6, pp.1119-1122. (In Chinese)

[9] F. Daowang, L. Teng and H. Z. Tao, Square-root second-order extended Kalman filter and its application in target motion analysis, in IET Radar, Sonar \& Navigation, Vol. 4 (2010) No. 3, pp.329-335.

[10]G.Q. He, X.K. Chen, Q.Y. Han et al. A Method of Automatically Improving SNR of Image[J]. Computer Technology And Development, Vol. 25 (2015) No. 12, pp.60-63. (In Chinese) 